parentibus femina certe satis nobilis et libera unb der Illiza ex utrisque parentibus femina nobilis et libera (Rang, Regeften 1, 91

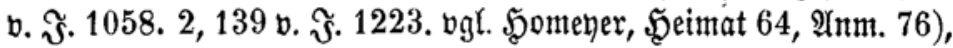

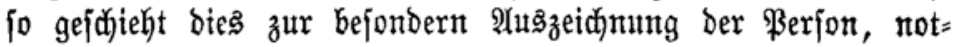
wentig aber war, wie idj a. a. $D .476$ f. Dargethan habe, eine

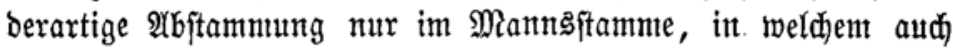

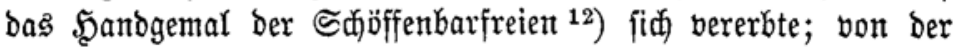

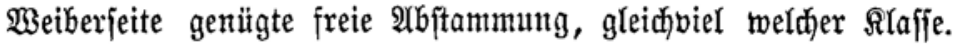
Anbers war bie Sache jajon nad Deutidjentp. 62: Es enist nieman gar vrei, wan des vater und des muter und der (๖. $\mathfrak{h}$. Deren) vater und der můter sentper vreien warn". ${ }^{13}$ ).

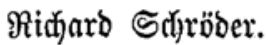

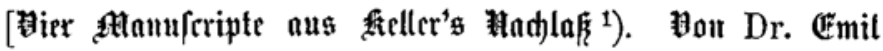
Steffenbagen, Frinatoocenter an der Wniver/ität šäniggberg.]

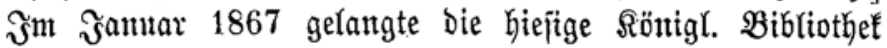

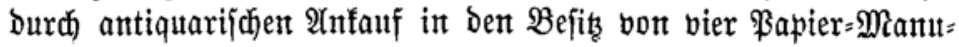
fcripten, bie beim exften cinblicf als herrïhrend aus ber ehemals

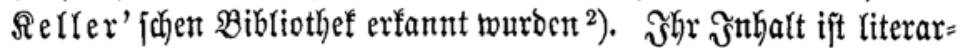

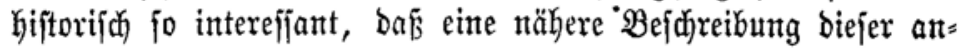
icheinend unbead)tet gebliebenen Mamujcripte geredtfertigt er= juheinent wirb.

I.

Das ältefte Manufcript, ein Folio= Bant aus bem Ento

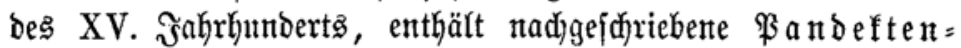

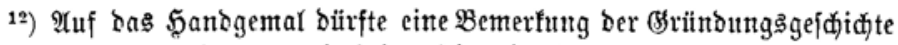
ses Stofters Baumburg zut bezichen feit, in ber es bon bem hodjebeln (Brafen Kunto heiß̄t: cuius genuinus et cognationis et posterorum eius postmodum communis locus usque hodie Uranthenhausen nuncupatur (Mon. boica 2, $173 \mathrm{ff}$.).

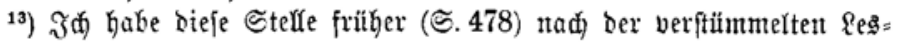

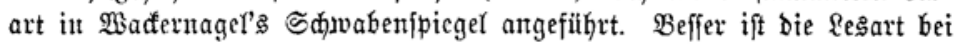
Paßjberg 70 b. But bergleident ift auth Rafiberg 123 a.

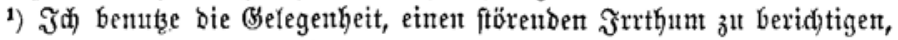

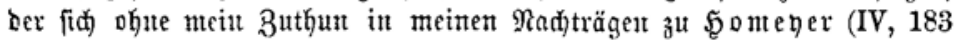

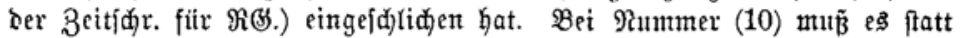

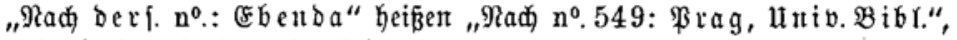

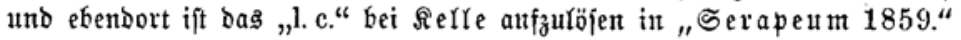

2) Sie tragen Refler's Wappen, mit ber Untidirift F. L. KELLER J. U. D. 
Borlejungen bes Safon vou Mayno, geharten zu Pavia

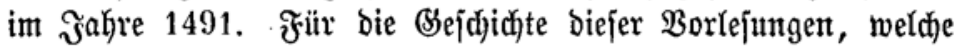

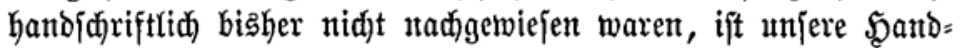

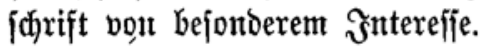

Die $\mathfrak{B o r l e j u n g e t t ~ v e r b r e i t e n ~ f i d ~ z u m a ̈ d ~ f j t ~ i n ~ f o r t l a u f e n t e r ~}$ Reihe über ben ritel De vulgari et pupillari substitutione $(28,6)$ von ber $\Re u b r i f$ und Der erften bis jur Yetzten Rex. Daran

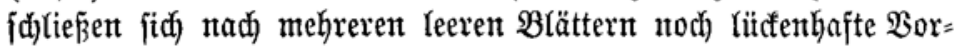
Yejuntgen über Rex 29 Gallus Aquilius des Titcle De liberis et postumis etc. $(28,2)$. Şiemit brich)t bie mudrfftäntige $\mathfrak{g}$. ab. Die ïbrigen $\mathfrak{B}$ lätter hat ber S(j)reiber Yeer gelaffent.

Die Bergleid)unt mit ber mir vorltegenden Drutfauggabe (I a s onis May ni Prima super Infortiato. Lugduni, 1540. fol.)

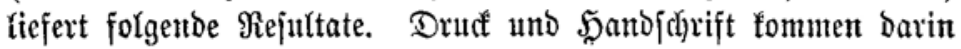
iïberein, Daß ber frühere Titel $(28,2)$ Dem fpäterent $(28,6)$ nach)=

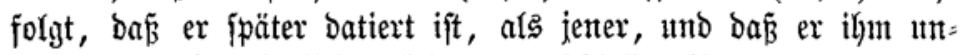

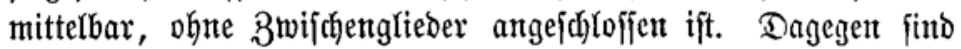
in Drute aud bie eriften 28 Leges Deș Titela 28, 2 behanteft, weldye in Der $\mathfrak{F}$. fehlent. Für Lex 29 beffelben Titeta liegent int

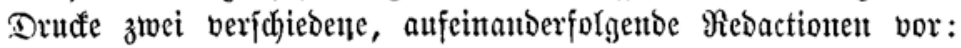

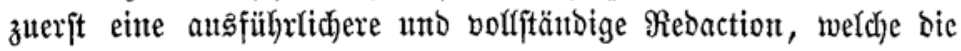
ganze Lex behandelt und ber Zeit nach fpäter fäflt; alsbantu eitte

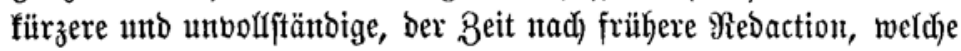
mit bem §. Instituens ${ }^{3}$ ) abfdjlief̧t und mit Der Borbenterkung verjehen ift: Sequitur l. gallus alias impressa. - Ista repe[titio] l. gallus fuit prima repetitio prefati domini Jasonis: sed non perfecta: alia fuit perfecta per ipsum. Diefe

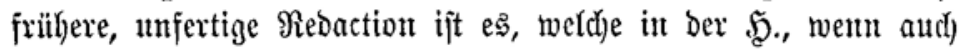

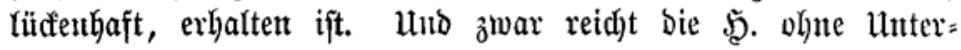
bredjung bis zu ben Worten: imo quod papa voluit solum committere, quod procedatur summarie gegent onbe von §. 2 Idem credendum (Col. 200" bes Drudes); hierauf fintoet fid

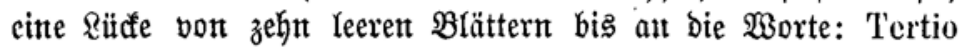
et forcius in contrarium facit, quia si vltra emancipacionem requireretur lapsus annj ïber §. 5 Et quid, si tantum (Col.

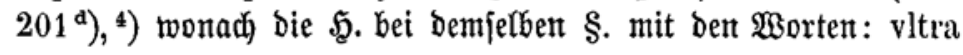

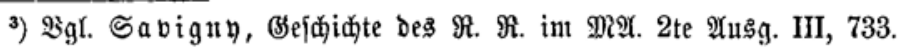
$\mathrm{n}^{0} .118$.

4) Die beident zrifdenfiegenden $\$ \$ .3,4$ fitto autd im Drưte nidht behandelt. 
concor[dantias] traditas per do. allex. (traditas) in istis locis

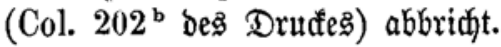

Borzüglid) beadftents?werth fint bie Data, welde in ber $\mathfrak{b}$. einzelnen $\mathfrak{B o r l e j u n g e n t}$ bei $=$ ober untergejdrieben fint. (Eines ber= jelbent (bas vierte) giebt zugleid) $\mathfrak{A}$ uffid)luf

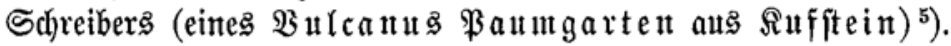

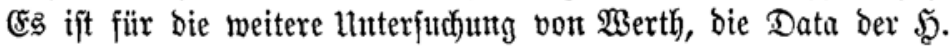
(im Sanzzen fünf) vollftändig mitzutheilen, und bautit bie Data bes Drufes, foweit fie vorhanben jint, z̆ vergleiden.

1) $3_{\mathfrak{u}} \mathfrak{A} \mathfrak{n} \mathfrak{n} \mathfrak{n} \mathfrak{t}$ bes $\mathfrak{T}$ itela 28,6 : De vulgari et pupillari substitutione Rubrica feliciter incipit. Lecta per do[minum] Jasonem de mayno in florentissimo gymnasio ticinensi, adi 23 del frebraro del 1491. (F̌bjit im Drute.)

2) $\mathfrak{A m}$ Schlnffe von Lex 15: Et per ista cum lande dei est impositus finis huic l. centurio in vigilia corporis $x \bar{p} i$, que fuit prima dies Junij 1491, in florentissimo gimnasio ticinensi per Jasonem de mainis. (Utebereinftimmeno im Druffe, Der nod hinzufïgt: et eam incepi die xj. Maij.)

3) $3 \mathfrak{t}$ Lex 45 princ.: Et per ista cum laude dei sit expedita ista reppeticio die antepe[nultima] $\mathrm{Julij}$ del 1491. (Fehtt im Drude.)

4) Sd) dei remanet expeditus presens titulus papie $\mathrm{dic}_{\mathrm{i}} \mathrm{iij}^{\mathrm{a}}$ mensis augusti del 1491 per excellentissimum d[ominum] Jaso. de maino. Et ego uulcanus paumgarten ex kuefstain scripsi sub codem, et non defficit sillaba. (Der Drud hat baffetbe Datum.)

5) Bei Lex 29 Iit. 28, 2: Inchoata est ista 1. foeliciter

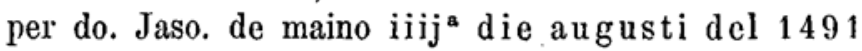

5) Diefer $\mathfrak{B}$ orfgang $\mathfrak{B a u m g a r t e n ~ a u s ~ b e m ~ b a n t a r s ~ n o d ~} B$ airifdjent

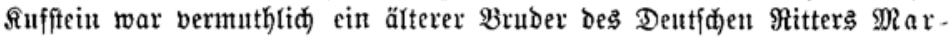
tiı von $\mathfrak{B}$ aumgarteł (Baumgartıer, Baumgärtner), welder Yețtere,

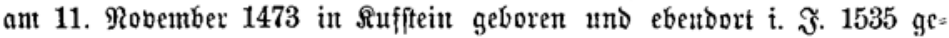
ftorbelt, Durd) feine Beziehungen zu \&uther, wie burd feine gebrutte "Peregrinatio in Aegyptum" etc. näber befaut ift (über ihn vgl. Sei be =

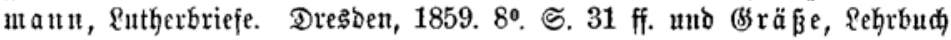
einer alfgem. \&iterärgefđ. III. 1. §. 322. ऽ. 1167). 
papie. (Frehlt in Druffe. Dagegen Datiert berjelbe bie

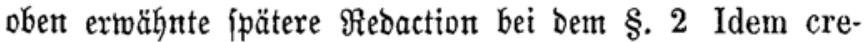
dendum in forgender $\mathfrak{B e i j e : ~ e t ~ c o n s e q u e n t e r ~ r e m a n e t ~}$ expeditus presens \&. die ij. iunij 1506 . intermissis tamen iamdudum quatuor lectionibus ob aduentum regis ${ }^{6}$ ) unt am Sabluffe ber ganzen \&ex: die xij. Augusti).

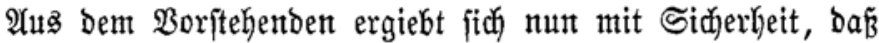

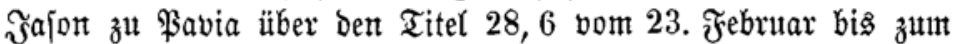

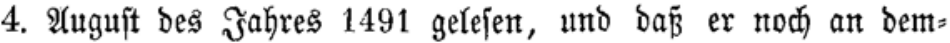
felbent Tage, an welchem ex ihn beendigte, bie Lex 29 bes Titela 28, 2 angefangen hat, unter Hebergehunt ber erften 28 Leges, weldye jich im Drude finten. Erft fpäter, unb zwar, wie bas Datum bes Drutces betweif́t, int $\Im .1506$ las er ben boflftänt= bigen Titel, und nodhmals bie Lex 29 in ber abmeidfenden Be: ftalt, wie fie im Drudfe an erfter Stefle vorliegt. Diejes Er:

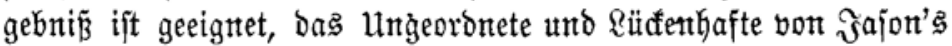
Borlejungen in ein ntues Ridjt zu jekzent. - Die brei lekztent I.eges $(30,31,32)$ von 28,2 idjeint $\mathfrak{J}$ afon ebentfalle nod) $i$. $\Im$.

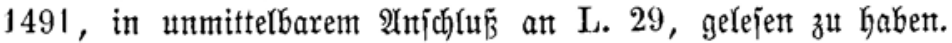
Denn, went am Entoe des Titels in bem vorliegenten Drute und ebenjo in edd. Lugd. 1546, Taurin. 1592 bas Datum fteft: die xiiij. Augusti, in ed. Lugd. 1582 blos: die xiiij, hingegen in edd. Venet. 1500 unt Mediol. 1508: MCCCCXCI. d. iiij. Aug. ( zahl 1491 fein Bebenfent erregen, unto ntur hintichttid bes Tages bleibt zweifelfaft, ob ber vierte ober ber vierzehnte alugujt anzunehmen fei.

Sabigny hat atts inneren (bründen gegen bie łahreszahl

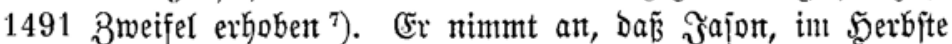

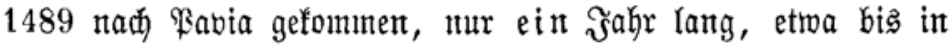
Dent Aruguft 1490 gelejent habe, worauf er, nadj feinem eigenten Beugntí (Consil. 93 Vol. III), über neunt Эahre burch eine Alugenidimäd)e verfindert wurbe, Borlejungen zu haltent. Dieje

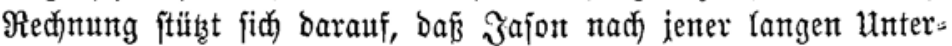
brechung auf Betreiben bes アöntigs \&ubwig XII. von Franfreid,

6) 2igl. atd Savigny l. c. VI. §. 113. e.

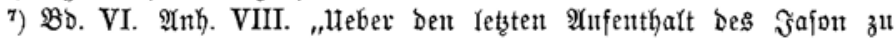
ßabia"; vgl. bazı §. 111. a, 113. f. h. 
weldjer Mailanto eroberte, feine Borlejungen wieder aufnabm, was in Spätjafr 1499 geidehen fein mußz. Sciemit jteht jebod unjere

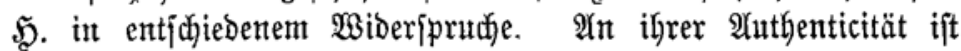

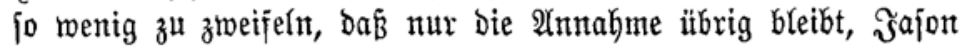
ferbft müffe jich geirrt haben, went er ben 3eitraum ber unter=

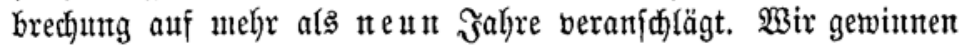

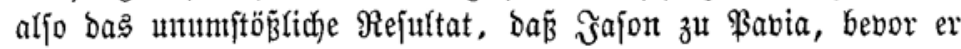
feine Borlejungen unterbrad, nidyt ein, fondern zwei Schul= jahre hindurd) (bis 1491) als \&ehrer thätig getwejen ift.

II.

Die brei ïbrigen Manujcripte (in @utart) gebören jänmtlich) in Den $\mathfrak{A}$ ugagang bes XVI. Jahrbuttoerts.

Das näd)jite Dem $2($ ter nad) bietet ein nod) unbefanntes Berf von B্s

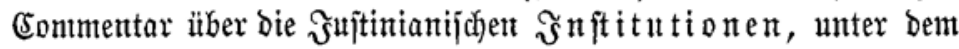
Titel: Commentaria in Libros Quatuor Institutionum Inperialium D. Iustiani [sic!] Sacratissimj Principis. Guidon is Pancirola e I. C. Clarissimi. Dag Âter bes MS. rejp. Der

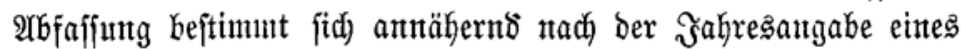
frïkeren Beïtzers auf bem zweiten (leeren) Blatte: Sum Matthaei Byttelmayrs Anno dni. 1. 5. 8. 7.

Pur wenig jünger, einander greidfzeitig uno, wie es fareint,

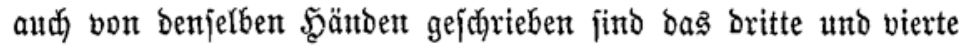
MS. Beibe liefern wertfodlfe Beiträge zur $\mathfrak{D e u t f d i e n , ~ w i e ~ b i e ~}$

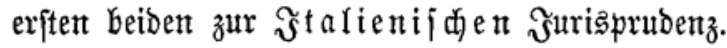

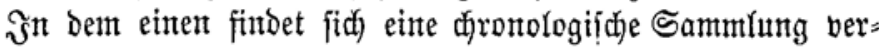

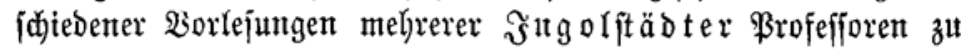
ben Bandeften, zur Auth. Novissima, unto zum Cobex; theiftweife mit Den Namen Der Berfaffer unt beigefügten Daten,

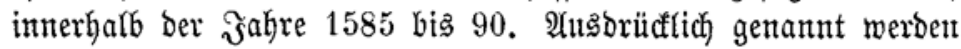

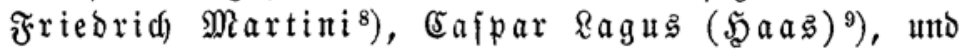

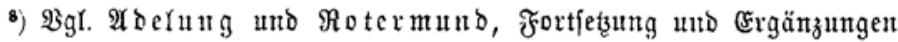

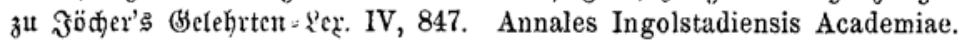
Inchoarunt Valent. Rotmarus et Io. Engerdus. Emendavit, auxit, continuavit . . I Io. Nepom. Mederer. Ingolstadii 1782. $4^{\circ}$. I, 294. II, $8,16,51,150$.

9) Adelug uto Rotermun 1. c. III, 1060. Annales Ingolst. Acad. I, 253. II, 3, 83, 186 f. 
Eafpar $\mathfrak{S} a ̈ \mathfrak{l l}$ (oder $\mathfrak{e} e \mathfrak{l l})^{10}$ ). $\mathfrak{O b}$ bie unbenantent Borlejun= gen bon einem ber Sentumten, ober bon 9lnberen herrühren, mut bahingeftellt bleiben. Ees wirb nicht überflitffig feit, bie ein= zetnen $\mathfrak{B o r l e f u n g e n t ~ z u ~ v e r z e i d n n e n t . ~}$

A. Friebrid Martini, 1585.

1) Repetitio L. vt vim [3] ff. De Iust. et Iure [1, 1], Clariss: doctissimiq. uirj Dn: Fridericj Martinj, in catholica Ingolst: Academia ordinarij professoris. - Auspicata 4. Febr. Anno 1585. Absoluta foeliciter 12. Iulij Anno 1585.

2) Explanatio L. Imperium [3] ff. De Iurisd: omnium Ind: $[2,1]$.

3) Interpretatio L. sciendum [15] ff. Qui satisdare cogantur $[2,8]$.

4) Dilucidatio L. Tres fratres [35] ff. de pactis $[2,14]$.

5) Explicatio L. Nesennius [34] ff. De negot:-gest: $[3,5]$.

6) Explicatio decretj D. Marcj in L. Extat [13] fr. de co quod metus caus: gest: erit $[4,2]$.

7) Interpretatio auth: Nouissima C. de inoffi: testa: $[3,28]$.

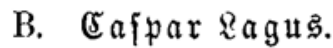

8) Repetitio in L. Nemo potest [55] ff. Ie Lega: $\mathrm{j}^{0}$ Clarissimi Doctissimique viri Dn: Caspari Lagi in Catholica Ingolsta: Academia ordinarij professoris.

9) Explicatio L. Finalis C. De Edicto Diui Hadriani tollendo $[6,33]$.

10) In L. 1. C. De Iure deliberandi $[6,30]$.

11) In L. 2. eod.

12) In L. 3. et L. Si infanti [18] eod.

13) In L. Cum in antiquioribus [19] cod.

14) In L. Finalem eod.

15) In L. 1. C. Qui admitti $[6,9]$.

16) In L. Cum quid [3] ff. Si cert: petatur $[12,1]$.

17) In L. Ciuitas [27] eod.

10) Annales Ingolst. Acad. II, 104, 192, 228. 
C. Cạpar $\mathfrak{b} a ̈ l l l(\mathfrak{l} e \mathfrak{l}), 1587$ bỉ 90.

18) In titulum C. communi diuidundo $[3,38]$ commentarius Clariss: doctissimique viri Dnj. Häll. I. V. D. et in catholica Ingolstadiana Academia ordinarij professoris. - L. 30. Iunij $\mathrm{A}^{0}$. Ini. 87, bis zum 12. September.

19) In titulum C. Finium Regundorum [3, 39]. Sout 14. bis iiber bent 23. Sept., ba bą leţ̧te Datum offen getaffent ift.

20) Interpretatio I. Qui Restituere [68] ff. De Rei vendicatione $[6,1]$ Clariss ${ }^{i}$ : viri Dn. Caspari Hell. I. V. D. ac in alma Ingolsta: Academia professoris ordinarij. - L. 8. Ianua: A. 90, bį über Den 5. F̌cbruar (bas leţ̧te Datım vacat).

21) Comentaria [sic!] in titulum ff. soluto matrimonio dos quemadmodum petatur [24, 3] clarissimi ac doctissimi viri Dn. Caspari Hell. I. V. D. ac professoris in alma Ingolsta: Academia ordinarij. - 2rngefangen vor Dem 29. Detober 1587. Finem imposuit huic materiae dotali die 9 Aprilis stylo nouo Anno 1588.

22) Shute Ueberidrift ein Dictat mit bem $\mathfrak{A n f a n g}:$ Exceptionis compensationis materiam explicandam desumpsimus. $\mathfrak{A} \mathfrak{n}$ zwei Stellett fint bie Data bei= gefügt: L. 9. Decemb: unt L. 16. Maij Anno 89;

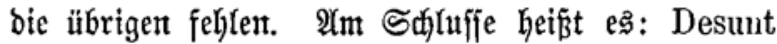
caetera et intacta relicta ab ipso dictando.

23) In L. si quis delegauerit [12] ff. De Nouatio: et Delegationib. $[46,2]$. - L. 4. Apri: $\mathrm{A}^{0} .90$, bį zum 5. Mpril. Mit biejer Borlejung endet Das vor= liegento MS.

Das leţte MS. endrid zeigt im alter und Eharafter ber Sajrift eine genaue Uebereinftimmung mit bem borhergehentont

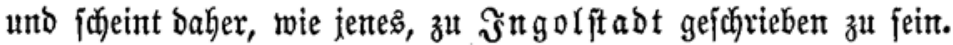
Darauf beutet aud vorn auf bem Dedel ber Rame Fachinacus (wohl ars bes $\mathfrak{B}$ efitzers ber $\mathfrak{5}$.$) , welcher um bie bamalige Beit$

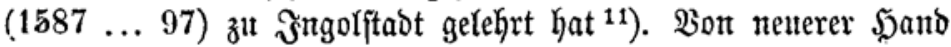

11) Annales Ingolstadiensis Academiae. II, 107, 148, 170. 


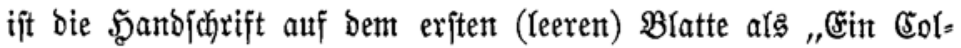
legieutheft proceffualifden Эnthalts" bezeidntet. Wir merben aucf bieje Borlejungent als $\mathfrak{s}$ golf̧t äbter anzujeben haben, nux fehit es an bem Ramen bes Berfajiers.

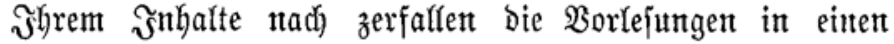
cibil=prozeffualifaen mo einen criminaliftifdeat Theil.

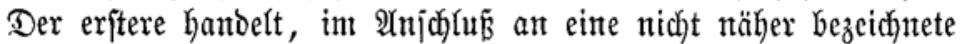
Stefle ber GSlofie, De Sententiis. Borangefchifft ift einte fitrze Sinteitung, welche nit Den $\mathfrak{B o r t e n}$ beginnt: Haec gloss: 3 habet

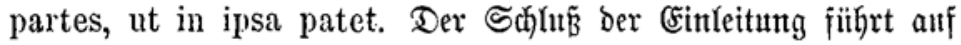
das Thenta uno bie Dispofition Des Branzan: Nunc autem occasione huius gl. materiam sententiarum plenius reassumemus constituendo titulum

\section{De Sententijs.}

Huius materiae tractationem in 3 partes principales distribuimus. In 1a de sententijs quaedam in genere dicemus. In 2 da specialiter de interlocutoria tractabinus. In 3 tia uero diffinitiua e sententiae materiam prosequemur. Der erfte Şaupttheil, De Sententijs in genere, wirb mur furz behandelt. Der zweite, De Sententia Interlocutoria, ift int 12 ungleiche Eapitel abgetheilt, mit ben Ueberichriften: 1) Interlocutoria Quid Sit. 2) In Quibus Sententia Interlocutoria differat à Diffinitiua. 3) Quot sint Interlocutoriae Sententiae species: et quae Sententiae pro interlocutorijs habeantur. 4) Qualiter Iudex super his, quae in Iudicio incidunt, uel emergunt sese gerere debeat. 5) Qualiter Interlocutoria sit formanda. 6) Qualiter et Quando Interlocutoria sit proferenda. 7) De effectibus Sententiae Interlocutoriae. 8) Quid in specie Iuris obtineat in decreto Iudicis. 9) Quid Iuris sit in praeceptis Iudicum. 10) Quid Iuris obtineat in Sententia mulctae. 11) Quid Iuris obtineat in Sententia excommunicationis, Interdicti, Banni, dispensationis, et alijs Similibus. 12) Quid Iuris obtineat In comminationibus, epistolis, programmatis, alijsque Similibus. Der britte unt lez̧te Şaupt= theif, De Sententijs Diffinitiuis, hat mur 4 Capitel, von Dentent

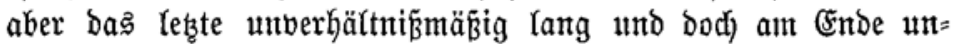
volrftänoig ift. Die Ucberfagriften lanten: 1) Quid sit diffinitiua: et quae sententiae pro diffinitiuis habeantur. 2) Quid 
Iudex circa diffinitiuam sententiam concipiendam, in genere obseruare debeat. 3) Quid In specie Iudici obseruandum sit circa probationes facti, ab alterutra, uel vtraque parte inductas. 4) Quid Iudex in specie obseruare debeat, circa facti probationem, quae interdum fit, per Iuramentum (in ber $\mathfrak{S}$.

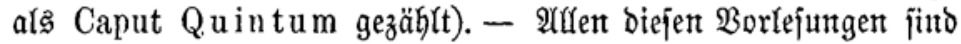
feine Datent beigejariebent, inteffen fint fie mit ben folgenden (batierter) angenifheinlid gleidgzeitig.

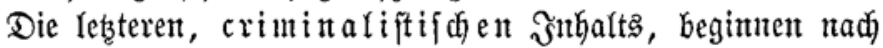

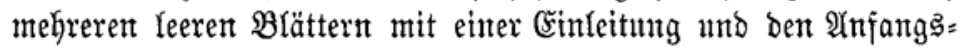
worten: Nulla re magis Reipublicae Status tranquillus conseruatur, quam si bonis praemia, malis uero poenae conlignae, respectu suorum delictorum tribuantur. Sie hanbeln über Den Criminal= Prozéz, und einleitend De officio Iudieis mit bent motivierentent $\mathfrak{L}$ ebergantge: Cum itaque intelligamus, quanti Reip: referat, bene moratos, doctos, ac eruditos iudices habere, ideo initio istius processus criminalis de officio eorundem dicemus. Die zahlreid) beigefügten Daten

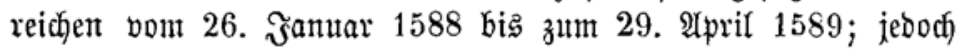

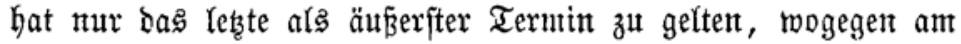

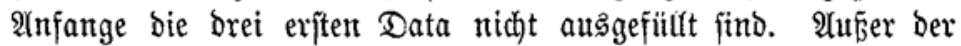
Cinleitung zerfallen bie Sorlejungen in 14 bald rängere, bals

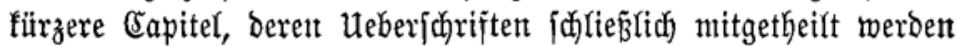
mögen: 1) De criminibus. 2) De Accusationibus. 3) De Demunciatione. 4) De exceptionibus criminalibus. 5) De Inquisitione. 6) De citatione. 7) De carceribus et carcerarijs. 8) De relaxatione carceratorum. 9) De exoninando ${ }^{12}$ ) siue excusando in processu criminali. 10) De publica fide seu salui conductu. 11) De libello seu petitione facienda in processu criminalj. 12) De quaestionibus et tortura. 13) De crimine lesae maiestatis diuinae et humanae (in ber $\mathfrak{H}$. ofne

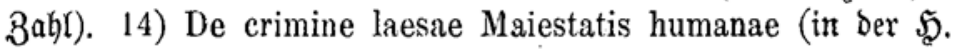
mit ber 3 ahl 11).

12) Ducange vocc. Exonia uno Sunnis. 
Brought to you by | New York University Bobst Library Technical Service Authenticated

Download Date | 6/8/15 12:23 PM 


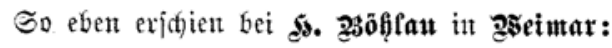

\section{$\mathfrak{A} \mathfrak{1} \mathfrak{\xi}$ \\ $\begin{array}{llllllllllllll}\mathfrak{A} & \mathfrak{D} & \mathfrak{m} & \mathfrak{u} & \mathfrak{n} & \mathfrak{d} & \mathfrak{B} & \mathfrak{y} & \mathfrak{z} & \mathfrak{a} & \mathfrak{n} & \mathfrak{z}\end{array}$ \\ $\mathfrak{y} \mathfrak{x} \mathfrak{t} \mathfrak{r} \mathfrak{i} \mathfrak{g} \mathfrak{e}$ \\ bolt}

ㄱ. $\mathfrak{D} \mathfrak{a} \mathfrak{n} \mathfrak{y}$,

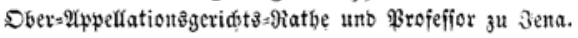

Frcis 28 ఠgr.

gुnfiaft:

(̌in Tag int römifiden Circus.

Bäber uno Babcleben in Rom.

Thuang unb Ende ber Bেlabiatorentämpfe.

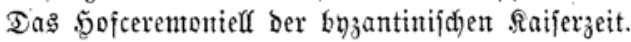

(sin 2Afruthr ber Farteien im Circus.

Verlag von Stifke t. van grutndent in Berlin.

Soeben erschien:

Kühns, Dr. Fr. Jul., Geschichte der Gerichtsverfassung in der Mark Brandenburg. 2 Bde. 55 Bog. Preis $5^{1 / 2}$ Thlr. 


\section{$\mathfrak{3} \mathfrak{n} \mathfrak{h} \mathfrak{a} \mathfrak{l} \mathfrak{t}$.}

Die Lex Maenia de dote bom Jahr ber Stabt $\Re$ om 568. Bon

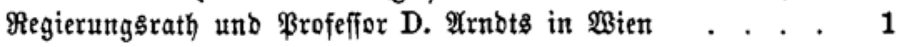

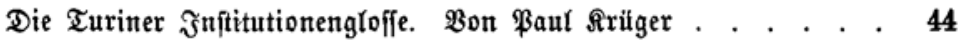
Heber bie Bebeutung beș Satzeş ber XII Tafeln Usus auctoritas fundi biennium, ceterarum rerum annus est, adversus hostem aeterna auctoritas. $30 n$ Profeffor D. Burđfharb in Jenta. .

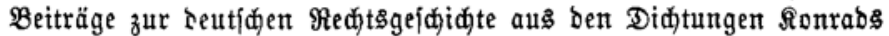

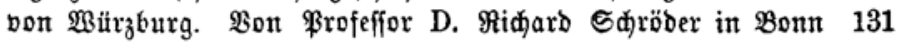
Misceller.

Siteratur. D. Seinrid Brunner, Zeugen und Эnquifitionşbe= weiș ber farolingifden Beit. Son $\Re u b o l p h$ Sohm in Böttingen 144

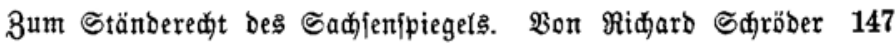

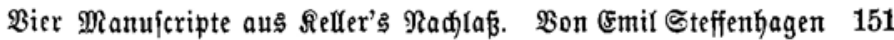

Auggegeben am 22 . Oetober 1867.

Weimax. - \$of = Buकbruderei. 